\title{
Whole-body MR and cysticercosis: reply to Wiwanitkit
}

\author{
Sheffali Gulati • Atin Kumar • \\ Ajit Harishkumar Goenka • Anita Choudhary • \\ Jitendra Kumar Sahu
}

Received: 23 March 2010 /Accepted: 23 March 2010/Published online: 1 May 2010

(C) Springer-Verlag 2010

We thank Dr. Wiwanitkit for interest expressed in our article [1].

Whole-body MR (WB-MR) is a recent development that refers to simultaneous whole-body coverage by acquiring multiple overlapping stations with the use of parallel acquisition technique (PAT) [2]. It is not the same as separate and non-contiguous conventional scanning of different body regions, which Dr. Wiwanitkit seems to have inferred. This distinction has been adequately emphasized in the article [1] and in the literature [2]. To the best of our knowledge, use of whole-body MR in the diagnosis of a disseminated parasitic disease has not been previously reported and hence, in our opinion, it is unique in the given scenario.

Hydatid cysts have been described in multiple locations besides liver. The unusual locations include lung, kidney, bone, brain, heart, spleen, pancreas, muscle, adrenal, ovary and peritoneum $[3,4]$. The imaging appearances of hydatid are distinctly different from cysticercosis, with the hydatid disease primarily consisting of large cysts (size usually of few centimeters to more than $15 \mathrm{~cm}$ ) that do not show any enhancement except for a thin rim of enhancement in few cases. Even when there is disseminated hydatid disease, the cysts are usually of large size and contain multiple small daughter cysts [5]. The muscle hydatids are extremely rare and have been reported as single or few conglomerate cysts, typically in one location [3-5]. In contrast, cysticercus cysts are typically very small, usually only a few millimeters. The cysticerci primarily occur in the brain or muscles. They are extremely unusual in liver and other solid abdominal organs, which are the usual sites of hydatid involvement. Such extensive involvement of the muscles of the whole body with involvement of practically every muscle fibre as seen in our case has been described in literature in disseminated cysticerci (but not by whole-body MRI) and has not been described in hydatid disease. In our patient, the differential of hydatid disease of disseminated cysticercosis is therefore inconceivable.

\section{References}

1. Kumar A, Goenka AH, Choudhary A et al (2010) Disseminated cysticercosis in a child: whole-body MR diagnosis with the use of parallel imaging. Pediatr Radiol 40:223-227

2. Kumar J, Seith A, Kumar A et al (2008) Whole-body MR imaging with the use of parallel imaging for detection of skeletal metastases in pediatric patients with small-cell neoplasms: comparison with skeletal scintigraphy and FDG PET/CT. Pediatr Radiol 38:953-962

3. Dahniya MH, Hanna RM, Ashebu S et al (2001) The imaging appearances of hydatid disease at some unusual sites. BJR 74:283-289

4. Ilica AT, Kocaoglu M, Zeybek N et al (2007) Extrahepatic abdominal hydatid disease caused by echinococcus granulosus: imaging findings. AJR 189:337-343

5. Polat P, Kantarci M, Alper F et al (2003) Hydatid disease from head to toe. Radiographics 23:475-494
S. Gulati $(\bowtie) \cdot$ A. Choudhary $\cdot$ J. K. Sahu

Department of Paediatrics, All India Institute of Medical Sciences, Ansari Nagar,

New Delhi 110029, India

e-mail: sheffaligulati@gmail.com

A. Kumar • A. H. Goenka

Department of Radiology, All India Institute of Medical Sciences, New Delhi, India 